\title{
Importance of Vascular Calcification in Kidney Transplant Recipients
}

\author{
Giuseppe Cianciolo Irene Capelli Maria Laura Angelini Chiara Valentini \\ Olga Baraldi Maria P. Scolari Sergio Stefoni
}

Nephrology, Dialysis and Transplantation Unit, Department of Experimental, Diagnostic and Specialty Medicine, S. Orsola University Hospital, Bologna, Italy

\section{Key Words}

Kidney transplant - Vascular calcification . CAC score .

Calcification inhibitors

\begin{abstract}
Background: Kidney transplantation is the treatment of choice for chronic kidney disease (CKD), but in kidney transplant recipients (KTRs) cardiovascular events are the first cause of death with a functioning graft, ranging from 36 to $55 \%$. The impact of vascular calcification (VC) on morbidity and mortality of KTRs is not appreciated enough nowadays. Summary: This review summarizes 13 important studies on VC in KTRs, comparing the results with CKD and dialysis populations. We focused on VC evaluation and use of coronary artery calcification (CAC) and aorta calcification (AoC) scores. We also evaluated the influence of traditional and non-traditional progression risk factors. Key Messages: VC strongly predicts cardiovascular events and all-cause mortality in KTRs. VC assessment is important in KTRs and based essentially on multislice computed tomography or electron beam computed tomography recognition of lesions. Quantitative measurement of CAC and AoC scores is essential for a correct definition of the calcium burden before and after kidney transplant. Progression of CAC slows down but does not halt
\end{abstract}

after kidney transplant. A variable association of both traditional and non-traditional risk factors is shown. There is a strong association between baseline CAC score and CAC progression. A significant improvement in secondary hyperparathyroidism after transplantation favorably affects the progression of CAC. Low $25(\mathrm{OH}) \mathrm{D}_{3}$ levels are an independent determinant of CAC progression. Diabetes is a risk factor for the presence of CAC in KTRs, but has not been independently associated with CAC progression. The data published on the use of immunosuppressive drugs as progression factors are few and inconclusive.

(c) 2014 S. Karger AG, Basel

\section{Cardiovascular Disease and Vascular Calcification in Kidney Transplant}

Cardiovascular disease (CVD) is the leading cause of death in kidney transplant recipients (KTRs), with a 3.5$5 \%$ annual risk of fatal or non-fatal cardiovascular events, much higher than in the general population despite adjustment for traditional risk factors. Death with graft function (DWGF) accounted for $42 \%$ of graft loss among KTRs: CVD is the most common cause of DWGF ranging from 36 to $55 \%[1,2]$.

\section{KARGER}

E-Mail karger@karger.com

www.karger.com/ajn
(C) 2014 S. Karger AG, Basel

0250-8095/14/0395-0418\$39.50/0
Prof. Sergio Stefoni

Dialysis, Nephrology and Transplantation Unit

S. Orsola University Hospital

via Massarenti 9, IT-40138 Bologna (Italy)

E-Mail sergio.stefoni@unibo.it 
There are three CVD presentations, namely coronary artery disease (CAD), left ventricular hypertrophy (LVH) and peripheral vascular disease. Except for $\mathrm{LVH}$, the main lesions underlying and ultimately responsible for the clinical manifestations are atheroma and vascular calcification (VC), which often co-exist. Calcification involves the entire vascular tree and is common in physiologic and pathologic conditions, i.e. aging patient, diabetes, dyslipidemia, genetic diseases, and principally in chronic kidney disease (CKD). In CKD patients, VC occurs both in the subintimal space (where it is typically associated with atherosclerotic disease) and in the media muscular layer of the arterial wall. Whereas intimal calcification is classically associated with advanced stages of atherosclerosis, calcification involving the muscular layer of medium-sized and large arteries is a process that is mainly linked with disorders of mineral metabolism and diabetes [3]. These two types of calcification can co-exist in the same vessel and be found near each other. Furthermore, intimal calcification seems to be more frequent than medial calcification and more extensive in patients on dialysis (particularly those with diabetes mellitus) than in individuals with normal renal function [4]. Even though intima and media calcification may vary in terms of location along the arterial tree (proximal vs. distal), clinical presentation and prognosis, in vivo studies in animal models show evidence for common pathomechanisms between VC and atherosclerosis and therefore ultimately between intima and media calcification [4].

In November 2013, we searched PubMed for original articles on VC in kidney transplant. This search produced 48 papers, among which we selected 13 pertinent papers. All papers were observational studies: 11 were prospective, 10 analyzed the prevalence or progression of $\mathrm{VC}$, and 3 studied the impact of VC and vascular outcomes. The prevalence of coronary artery calcification (CAC) in KTRs is higher $(61-75 \%)$ than that assessed in stage 3 CKD [5-7] and lower than that found in hemodialysis patients [8], possibly due to a selection bias of KTRs upon admission to the waiting list. The determinants associated with its presence are: age, dialysis vintage, time on transplantation and high/low turnover bone disease [9].

As in CKD patients $[10,11]$, KTRs' VC strongly predicts cardiovascular events and all-cause mortality over conventional risk factors. Several imaging methods are currently available to investigate $\mathrm{VC}$, such as cardiac computed tomography (CT), planar X-ray, echocardiography and vascular ultrasound. Even using the most highly performing imaging techniques presently available, it is impossible to distinguish between intimal and medial calcification. By contrast, non-invasive imaging methods have the potential to distinguish patients at increased risk of future cardiovascular events and mortality from those in whom the absence of VC may imply better prognosis. Electron beam computed tomography (EBCT) and multislice computed tomography (MSCT) are well-validated, non-invasive imaging methods and are considered the standard reference for assessing CAC, aortic and valvular calcification; they also carry no requirement for contrast administration. Scores such as the Agatston score, volume score and mass score are used for quantification of VC. The volume and mass scores are quantitative and more reproducible measurements $\left(\mathrm{mm}^{3}\right.$ or $\mathrm{mg}$, respectively), in addition to being more appropriate for use with modern CT scanners than the Agatston score. However, the Agatston score (semiquantitative) is the most frequently used and reported method in medical literature. The presence and extent of CAC, occurring only within the setting of atherosclerosis, correlates with the overall magnitude of the atherosclerotic plaque burden, severity of stenosis and myocardial ischemia [12].

Similarly, any single isolated calcium deposit found should be considered as an index for possible presence of coronary disease. Again, aortic and valvular calcification can be assessed by CT methods and has been associated with mortality and cardiovascular events in non-renal and CKD patients [13, 14].

\section{Vascular Calcification Progression and Cardiovascular Outcomes}

The methods and the results of the studies focused on CAC progression and cardiovascular outcomes are not uniform (table 1). Early studies performed with short intervals between CT assessments suggested that progression of CAC slows down or stops following an early phase of progression. In a small study on 31 patients, Oschatz et al. [15] observed a significant progression within the first 6 months, but no significant change between months 6 and 12 after kidney transplant. In contrast, Moe and Chen [3] did not observe CAC progression and Bargnoux et al. [16] observed CAC progression in $26.3 \%$ of their patients.

Further studies have evaluated CAC progression in renal transplant recipients in a longer follow-up. Mazzaferro et al. [17] compared 2-year CAC changes in 41 prevalent KTRs for at least 6 months and in 30 dialyzed patients: the progression was observed in $12.2 \%$ of the KTRs. The authors concluded that renal transplantation favor- 
Table 1. The results of the largest studies on progression and cardiovascular outcome of VC after kidney transplant

\begin{tabular}{|c|c|c|c|c|c|}
\hline Study & Objective & $\begin{array}{l}\text { Participants, } \\
\mathrm{n}\end{array}$ & $\begin{array}{l}\text { Follow-up, } \\
\text { years }\end{array}$ & Progression & Risk factor \\
\hline $\begin{array}{l}\text { Maréchal et al., } \\
2012 \text { [19] }\end{array}$ & $\begin{array}{l}\text { to assess the evolution of } \\
\text { CAC and thoracic AoC }\end{array}$ & 197 & $4.40 \pm 0.28$ & $\begin{array}{l}\text { CAC and AoC scores (mg) } \\
\text { increased by a median of } 11 \\
\text { and } 4 \% \text { per year, respectively }\end{array}$ & $\begin{array}{l}\text { higher baseline CAC score, } \\
\text { history of cardiovascular event, } \\
\text { use of a statin, and lower } \\
25(\mathrm{OH}) \mathrm{D}_{3} \text { level }\end{array}$ \\
\hline $\begin{array}{l}\text { Seyahi et al., } \\
2012[18]\end{array}$ & $\begin{array}{l}\text { to determine the } \\
\text { progression of CAC }\end{array}$ & 150 & $2.8 \pm 0.4$ & $\begin{array}{l}\text { CAC prevalence increased to } \\
64.6 \% \text { and mean CAC score } \\
\text { to } 94.9 \pm 245.7\end{array}$ & $\begin{array}{l}\text { baseline CAC, high triglyceride } \\
\text { and bisphosphonate use }\end{array}$ \\
\hline $\begin{array}{l}\text { Nguyen et al., } \\
2010 \text { [64] }\end{array}$ & $\begin{array}{l}\text { to assess the prognostic } \\
\text { impact of CAC on } \\
\text { cardiovascular event }\end{array}$ & 281 & $2.3 \pm 0.5$ & $\begin{array}{l}16 \text { patients died from } \\
\text { cardiovascular or non- } \\
\text { cardiovascular causes; } \\
31 \text { patients developed at least } \\
\text { one cardiovascular event }\end{array}$ & CAC score \\
\hline $\begin{array}{l}\text { Bargnoux et al., } \\
2009 \text { [16] }\end{array}$ & $\begin{array}{l}\text { to assess the evolution of } \\
\text { CAC }\end{array}$ & 83 & 1 & $\begin{array}{l}\text { CAC progressed in } 26.3 \% \text { of } \\
\text { patients }\end{array}$ & baseline CAC \\
\hline $\begin{array}{l}\text { Oschatz et al., } \\
2006[15]\end{array}$ & $\begin{array}{l}\text { to measure the extent of } \\
\text { CAC }\end{array}$ & 31 & 1 & $\begin{array}{l}\text { mean total Agatston score } \\
\text { increased significantly from } \\
\text { baseline to } 6 \text { months } \\
(716 \pm 980 \text { vs. } 916 \pm 1,307)\end{array}$ & $\begin{array}{l}\text { duration of pre-transplantation } \\
\text { dialysis treatment and smoking }\end{array}$ \\
\hline $\begin{array}{l}\text { Schankel et al., } \\
2007 \text { [23] }\end{array}$ & $\begin{array}{l}\text { to determine the natural } \\
\text { history and risk factors } \\
\text { associated with CAC }\end{array}$ & 82 & 1 & $\begin{array}{l}\text { CAC score increased for all } \\
\text { subjects; mean annualized } \\
\text { rate of CAC score change } \\
\text { was } 52.5\end{array}$ & $\begin{array}{l}\text { diastolic blood pressure, } \\
\text { Caucasian race, glomerular } \\
\text { filtration rate at } 3 \text { months post- } \\
\text { transplant, BMI, CAC score }\end{array}$ \\
\hline
\end{tabular}

ESR $=$ Erythrocyte sedimentation rate.

ably affects but does not halt CAC progression. When Seyahi et al. [18] studied CAC progression in 150 prevalent KTRs without documented CVD, the median followup time was 2.83 years. In patients with baseline CAC, the median annualized rate of CAC progression was $11.1 \%$, while in patients without CAC at baseline the yearly incidence rate of CAC was $12.5 \%$.

The study by Maréchal et al. [19] was the first to assess progression of both CAC and aorta calcification (AoC) in a large population of stable KTRs with a relatively long follow-up. Indeed, it was the only that evaluated CAC progression with a quantitative method. Their major finding is that VC progresses substantially within 4 years in prevalent KTRs: CAC increased by a median of $11 \%$ per year and $\mathrm{AoC}$ increased by a median of $4 \%$ per year. In $25 \%$ of patients, the yearly increase in CAC and AoC was 23 and $17 \%$, respectively. The authors did not assess CAC incidence but found that among patients with an initial CAC score of $0 \mathrm{mg}$, a total of $83.3 \%$ once again had a score of $0 \mathrm{mg}$. These discrepancies in CAC progression on KTRs could be partially related to demographic and clinical differences of the study populations, but there are some other variables that should be considered in CAC progression studies: (1) interscan variability of CAC scoring; (2) a CAC score reflects the aggregate of medial and intimal wall calcification: a different progression pattern can be expected for each type of calcification, and (3) the optimal time interval for measuring CAC progression is not defined. For the purpose of interventional trials, intervals of at least 3 years were proposed [20]. The time interval for measuring CAC progression is critical in transplantation where the risk factors relating to it start at the time of surgery. In two different studies $[18,21]$, each additional year of followup was associated with an average extra 17.5 Agatston units of progression. Furthermore, the need for follow- 
up may be different, depending on the CAC baseline score: in patients with a baseline CAC score of 0 , progression of CAC seems non-linear and extremely low in the first years, so the assumption that there is a constant rate of CAC progression might be incorrect since there is a lag period in which an individual without CAC may show no progression [18].

Even with the above limitations and despite there being no control group available in the studies made on KTRs, the comparison with two larges studies can provide useful data to analyze kidney transplant comparatively with both non-renal and HD patients in relation to the yearly incidence rate of CAC. In KTRs the CAC incidence rate is similar to that found in the Multi-Ethnic Study of Atherosclerosis which ranges from 5\% annually in those $<50$ years of age to $12 \%$ in those $>80$ years of age [21], while it is significantly lower than those found in HD patients [22]. In KTRs, partly due to the calcification burden of the dialysis period, CAC progression is a quite usual process, although at a rate significantly lower than that of HD patients [20, 22]. However, in two studies [19, 23], $25 \%$ of patients showed a yearly increase in CAC $>23 \%$ versus a median of $11 \%$.

Regarding AoC prevalence and progression, the data for KTRs are limited. DeLoach et al. [24] reported a prevalence of $34 \%$ in a cohort of incident transplant recipients and demonstrated that aortic calcification is predictive of cardiovascular events. Moe et al. [25] showed no significant progression of AoC, probably due to a small cohort and short follow-up. Maréchal et al. [19] for the first time observed in KTRs that there is a positive correlation between absolute annualized progression of CAC and AoC; moreover, AoC increased by a median of $4 \%$ per year. Previous studies have shown the association between CAD and AoC using EBCT [26, 27]. Adler et al. [28] not only found by spiral CT that there was a significant association between presence of CAC and calcification of the thoracic aorta but also that CAC is such a strong marker of AoC that it eliminated most conventional risk factors for atherosclerosis in multivariate analyses. The association of CAC with AoC leads to some considerations. Unlike the atherosclerotic nature of $\mathrm{CAC}, \mathrm{AoC}$ is an expression of both intima and media calcification. However, it must be emphasized that CAC and AoC often co-exist, besides having a partially overlapping pathogenetic basis (namely, aging and diabetes), so combination of the two may express the same risk burden.

Many findings suggest that progression of VC in KTRs is not homogeneous and is influenced by several factors, some of which are unknown.

Vascular Calcification in Kidney

Transplant

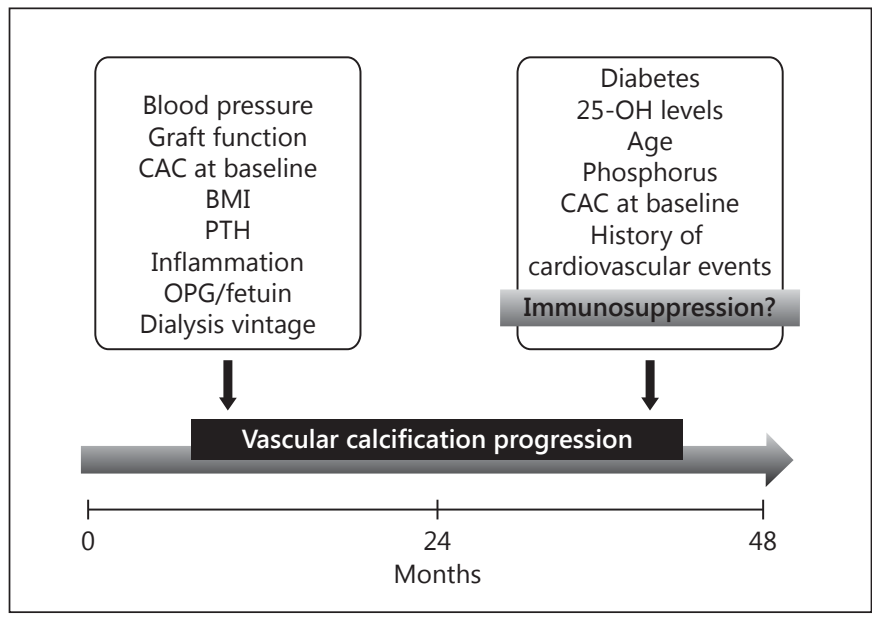

Fig. 1. Modification of risk factors for the progression of VC in kidney transplant patients during the time following transplant.

\section{Progression Risk Factors}

One unanswered problem, in both non-renal patients and $\mathrm{CKD} / \mathrm{KTRs}$, is why CAC progresses more in some patients than in others. The few studies made on KTRs, focusing on CAC progression, have shown a variable association of both traditional (blood pressure, smoking, dyslipidemia, age, body mass index and history of cardiovascular events) and non-traditional risk factors (CAC at baseline, inflammation, hyperparathyroidism and dialysis duration) (fig. 1) [18, 29-31]. The strong association between baseline CAC score and CAC progression confirms previous studies on non-renal and hemodialysis patients [22]. The patients with CAC at baseline probably came from a worse metabolic and inflammatory situation and inherited a more severe vascular disease from the pre-transplant period. In light of the strong link of CAC (most likely related to the extent of atherosclerosis) with a history of cardiovascular events in both ESRD and KTRs $[9,22,23]$, it is foreseeable that a history of cardiovascular events may predict CAC progression [19].

\section{Mineral Metabolism}

While in ESRD patients CAC is mainly associated with chronic disturbances of the mineral metabolism and inflammation [32], in KTRs the association of CAC with the main CKD-mineral and bone disorder (CKD-MBD) mediators is less evident. Mazzaferro et al. [29], after an interval of 2 years, detected a significant improvement in the biochemical parameters of secondary hyperparathy- 
roidism in transplant patients which, as expected, favorably affected the progression of CAC. A low $25(\mathrm{OH}) \mathrm{D}_{3}$ level is an independent determinant of CAC progression in KTRs $[9,19]$. In addition to its role in mineral and bone metabolism, vitamin D has many other pleiotropic effects. Several studies have shown that vitamin D deficiency is associated with increased risk of CVD and cardiovascular mortality whether in the general population [32, 33], in CKD or in hemodialysis patients.

Previous studies observed a negative correlation between vitamin D levels and endothelial function [34]. These findings support the hypothesis that vitamin D deficiency is associated with endothelial dysfunction, a prerequisite of both atherosclerosis and arteriosclerosis and hence of intima and medial calcification [35]. Currently no intervention studies have demonstrated the efficacy of vitamin $\mathrm{D}$ therapy in the prevention or treatment of $\mathrm{VC}$ in kidney transplantation.

Hyperphosphatemia has been associated with the prevalence and progression of $\mathrm{VC}$ in $\mathrm{CKD}$ and dialysis patients $[36,37]$. Phosphate directly induces a calcifying phenotype in vascular smooth muscle cells as well as endothelial dysfunction and intima calcification [38, 39]. However, although Connolly et al. [40] demonstrated that serum phosphate levels were significantly associated with mortality, none of the prospective studies carried out in these patients showed a significant association between CAC progression and phosphate levels. Only one study [19], using a multivariable regression model, identified that phosphate levels are independent determinants of aortic calcification progression. One possible explanation for these contradictory results may lie in the normalization of phosphate values after transplantation. However, despite fibroblast growth factor 23 (FGF-23) being the principal regulator of phosphate handling, its possible role on VC is still an underexplored area of investigation in KTRs. In CKD patients, FGF-23 levels are strongly associated with mortality, regardless of the phosphate concentration [41], and have been surmised to play a role in LVH, endothelial function, CAC and atherosclerosis [42]. In KTRs, high FGF-23 levels may persist for a long time, partly as consequence of steroid therapy and parathormone (PTH) [43]. In a study on 984 prevalent KTRs, elevated FGF-23 levels were independently associated with greater subsequent risks of mortality and allograft loss [44].

\section{Calcification Inhibitors}

Despite the powerful pro-calcification effect of the above-mentioned factors, some patients do not develop CAC and it rarely develops in follow-up.
In previous studies on non-renal patients with no CAC at baseline [20], no CAC progression developed in 62$75 \%$ of patients. In a study on KTRs, $65 \%$ of 97 patients with no baseline CAC did not convert to a positive score after 2.8 years [18]. Among selected patients Maréchal et al. [19] found a CAC score of 0 in $24.4 \%$ and an AoC score of 0 in $18 \%$. Respectively $83.3 \%$ (40) and $75 \%$ (27) still had a score of 0 after 4 years of follow-up. We have no exact explanations for patients who remain free of $\mathrm{VC}$ in the follow-up, but we can assume that such patients may be genetically protected or have high levels of calcification inhibitors or both (see below).

There are few data about changes to the proteins involved in VC in KTRs, although some authors [29, 45] have reported a trend toward normalization for serum levels of calcification inhibitors after transplantation: fetuin-A, matrix Gla protein (MGP) and osteoprotegerin (OPG). Fetuin-A serum levels are responsible for approximately $50 \%$ of the calcification inhibitory capacity of normal human plasma, so higher values are expected to be protective for calcification. MGP exerts its inhibitory effect at the tissue level after vitamin K-dependent $\gamma$-carboxylation activation. OPG, mainly produced by osteoblasts and cardiovascular cells, is a decoy receptor activator for the receptor activator of nuclear factor- $\mathrm{kB}$ ligand (RANKL). By binding RANKL and preventing the interaction with RANK, OPG inhibits the differentiation of osteoclast precursors into mature osteoclasts. The same effect takes place on VC, where OPG hinders the differentiation of monocytes/macrophages into an osteoclast-like cell phenotype favoring the prevalence of osteoblast-like cells and thus the progression of VC.

The fetuin-A reduction has been related to the presence of a lower degree of inflammation in KTRs. Maréchal et al. [19] showed that low fetuin-A levels are independently associated with $\mathrm{AoC}$ and predict a higher risk of long-term cardiovascular events and deaths in KTRs. The risk related to low fetuin-A is influenced by inflammation, while fetuin-A levels are determined by a common haplotype of the $\alpha_{2}$-Heremans-Schmid glycoprotein as well as by low plasma cholesterol and a history of smoking.

There are at least two variables that can affect the levels of MGP activity. Yoshikawa et al. [46] assessed that polymorphism in the MGP gene is associated with the progression of VC in HD patients. Low vitamin $\mathrm{K}$ status due to poor intake or the use of vitamin $\mathrm{K}$ antagonists results in high uncarboxylated MGP (ucMGP) levels and is associated with VC in all populations. The plasma desphospho-ucMGP (dp-ucMGP) fraction is considered a mark- 
er for vascular vitamin $\mathrm{K}$ status [47]. However in their study, Mazzaferro et al. [29] did not differentiate between carboxylated and unMGP. Boxma et al. [48] found that insufficient vitamin $\mathrm{K}$ intake and vascular vitamin $\mathrm{K}$ insufficiency (deduced from circulating dp-ucMGP levels) were very common in a population of stable KTRs and identified warfarin use as an independent determinant of dp-ucMGP levels. All these findings lead one to believe there is an association between use of anticoagulant drugs and VC. However, no study has so far verified the effect of vitamin $\mathrm{K}$ therapy in this population. In non-renal and renal patients, increased levels of OPG are associated with all-cause mortality and cardiovascular outcome in both HD patients [49] and KTRs [50]. The increase in OPG could follow on the fact that it is simultaneously being synthesized in vessel walls by activated osteoblast-like cells responsible for calcification.

The improvement in CKD-MBD, along with normalization of calcification inhibitory proteins following successful transplantation, could be a possible key to understanding the reduced progression of CAC in KTRs compared to HD patients [24, 29].

\section{Diabetes}

Diabetes is a known predictor of CAC in KTRs $[9,30]$, but has not been independently associated with CAC progression in any of the prospective studies conducted so far. Mehrotra et al. [31] found that diabetics with nephropathy were more likely to have progressive calcifications when compared with normoalbuminuric diabetic subjects. Schankel et al. [23] hypothesized that much of the effect of diabetes on CAC is mediated by renal function, which was improved in this cohort after renal transplant. Other studies that have shown progression of CAC in diabetics failed to adjust for renal function measurements [51]. Moreover, they were unable to find any difference in glucose levels between progressors and nonprogressors at the time of transplant or at 3 months. Suboptimal glycemic control $\left(\mathrm{HbA}_{1 \mathrm{c}}>7.5 \%\right)$ was found to be a strong risk factor for progression of CAC in a cohort of 109 type 1 diabetics [52]; hyperglycemia has been found to directly induce phenotypic change of vascular smooth muscle cells to osteoblast-like cells with increased bone matrix protein expression [53]. Unfortunately, in other prospective studies made on KTRs no information on adequate glycemic control during follow-up was included.

\section{Drugs}

Despite the important role that immunosuppressive therapy plays in graft and patient survival, few studies

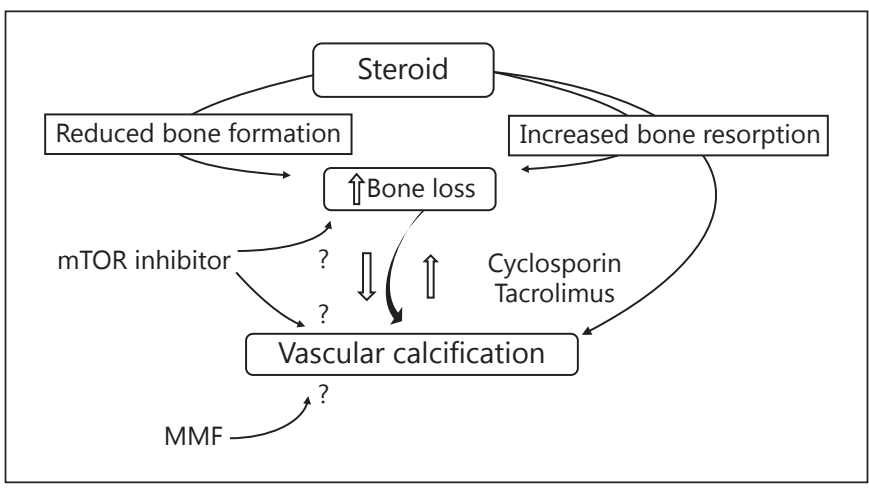

Fig. 2. Role of immunosuppressive drugs in post-transplant bone disease, the development and progression of VC in kidney transplant patients.

have analyzed its impact on the incidence and progression of VC (fig. 2). Notwithstanding this, cardiovascular risk factors directly or less directly related to immunosuppressive drugs, such as hyperlipidemia, hypertension, diabetes, and endothelial dysfunction, tend to be associated with VC. Proliferation inhibitors such as mycophenolate mofetil (MMF) have a more favorable effect on endothelial activity than do calcineurin inhibitors and steroids [54].

Nguyen et al. [9] found that a longer time spent under MMF was protective against aortic calcification, while experimental studies have demonstrated that MMF inhibits vascular smooth muscle cell proliferation in rats [55]. Steroids and calcineurin inhibitors inhibit inducible nitric oxide, thus helping to determine endothelial dysfunction associated with onset and progression of atherosclerosis and VC. Much more complex are the vascular effects of mTOR inhibitors. Rapamycin inhibits smooth muscle cell proliferation, while everolimus impairs the vasoactive and antithrombotic function of endothelial cells. Some studies suggest a relationship between $\mathrm{VC}$ and impaired bone metabolism as well as an involvement of immunosuppressive drugs on expression, regulation, and function of RANKL, RANK, and OPG system working in skeletal and vascular systems. In particular, sirolimus inhibits osteoclast formation, unlike steroids and cyclosporine [56].

Hence, the hypothetical effect of immunosuppressive therapy on VC could be mediated indirectly, by interfering with post-transplant bone loss, and directly through effects that the same drugs exert on vascular cells (endothelial cells, vascular smooth muscle cells) through intermediation of the RANKL, RANK, and OPG systems. 
Maréchal et al. [19] argued that statin treatment is predictive of CAC and AoC progression in KTRs. The argument may, of course, be circular, patients with adverse risk profiles being more likely to be on statin therapy. However, there are other interpretations of this result. While it is possible to induce regression of the portion of non-calcified plaque with statin therapy, it is unknown whether this is possible with the portion of calcified plaque. Pathologically, statins have been shown to promote microcalcification, which might increase the CAC score even though total atherosclerosis is reduced on statin therapy [57]. Moreover, when statins reduce the soft lipid core of a calcified plaque, the density of the plaque and its Agatston calcium score might increase, whereas its volume might decrease.

\section{CAC Assessment}

There is an ongoing debate on the use of CAC scoring as a screening tool since the presence of calcium in coronary arteries is pathognomonic of atherosclerosis. $\mathrm{CAC}$, detected and quantified by $\mathrm{CT}$, affords a reliable linear anatomic estimate of the total plaque burden and is taken clinically as a 'calcium score'. In the general population, the presence of CAC is associated with symptoms of coronary ischemia and there is a trend towards higher rates of coronary ischemia by scintigraphy in patients with severe CAC $[5,58]$. In these studies the prevalence of silent myocardial ischemia by scintigraphy was found to be between 10.4 and $46 \%$ in patients who had a CAC score $>400$. Since CAC scores are higher in transplant recipients, it is reasonable to say that a higher ( $>400)$ CAC score cut-off level should be used before asymptomatic candidates for renal transplantation are screened for myocardial ischemia. Numerous studies indicate that the CAC score correlates with the number and severity of conventional risk factors but is also an independent marker for the risk of coronary events, after adjustment for those conventional risk factors $[59,60]$.

Nearly all prospective studies have found that not only should conventional risk factors and the Framingham risk score be considered as predictors of future cardiovascular events, but that even moderate-to-high CAC should also be considered as an independent and incremental predictor of future cardiovascular events. Several studies carried out on dialysis patients and KTRs, as well as in the general population, have shown a strong association between a history of cardiovascular events and mortality with CAC [6]. Despite this, there is no clear indication for including non-contrast CT in the instrumental diagnostic procedure to detect the presence and entity of the calcium load and hence also subclinical atherosclerosis in kidney transplantation candidates and KTRs.

The 2010 ACCF/AHA Guidelines for Assessment of Cardiovascular Risk consider measurement of CAC for cardiovascular risk reasonable in asymptomatic patients at intermediate and low-to-intermediate risk. On the other hand, the 2009 KDIGO guidelines state that the KTRs must be considered high-cardiovascular-risk patients [61]. Unexpectedly, a scientific statement by the same society spoke of being uncertain how useful CT calcium scoring might be for the assessment of pre-transplantation cardiovascular risk [62]. A rational approach here could be to integrate the CAC score with that of the Framingham risk score. Degoma et al. [63] demonstrated that the incorporation of low-, high- and very-high-risk CAC values using the MESA risk score resulted in greater changes in the absolute risk of CAC as a predictor of mortality.

\section{Conclusions}

In KTRs, VC is to some extent a legacy. The burden of risk factors present before transplantation is bound to worsen during the phase of ESRD, as well as being conditioned by demographic and clinical patient characteristics. The measurement of CAC and AoC scores is essential for a correct definition of the calcium burden before kidney transplantation, and possibly incorporating it in comprehensive models of other CVD risks. Kidney transplantation slows, but does not halt, the progression of both CAC and AoC compared to the pattern observed in hemodialysis patients. Is not clear from the literature review what causes this reduction. We can only assume that the regression of the uremic milieu plays a fundamental role. The progression of $\mathrm{VC}$ after renal transplantation is conditioned by the calcification score at baseline and by the action of Framingham factors (dyslipidemia, blood pressure, smoking). The role of diabetes is not clear in the studies analyzed. CKD-MBD improvement has a positive influence on the calcification progression rate. Reduced fetuin-A levels and reduced activity of MGP are found in KTRs with VC. We are not able to determine whether and what role immunosuppressive therapy may play either through a direct action on the vascular wall or indirectly through effects on the process of bone remodeling. In ad- 
dition, we still do not know why a subset of patients should have zero calcification score, nor have 0 CAC patient characteristics been well described. However, we can assume that such patients may be genetically protected or have high levels of calcification inhibitors, or both. We believe further studies are needed, focusing on the impact of these last points.

\section{Acknowledgment}

The authors are grateful to Helen Spiby for editorial assistance.

\section{Disclosure Statement}

The authors have no conflicts of interest to disclose.

\section{References}

1 Ojo AO, Morales JM, González-Molina M, et al: Comparison of the long-term outcomes of kidney transplantation: USA versus Spain. Nephrol Dial Transplant 2013;28:213-220.

2 Kahwaji J, Bunnapradist S, Hsu JW, Idroos ML, Dudek R: Cause of death with graft renal function among renal transplant recipients in an integrated healthcare system. Transplantation 2011;91:225-230.

3 Moe SM, Chen NX: Mechanisms of vascular calcification in chronic kidney disease. J Am Soc Nephrol 2008;19:213-2162.

4 Nakamura S, Ishibashi-Ueda H, Niizuma S, Yoshihara F, Horio T, Kawano Y: Coronary calcification in patients with chronic kidney disease and coronary artery disease. Clin J Am Soc Nephrol 2009;4:1892-1900.

$\checkmark 5$ Seyahi N, Kahveci A, Cebi D, et al: Coronary artery calcification and coronary ischaemia in renal transplant recipients. Nephrol Dial Transplant 2011;26:720-726.

6 Maréchal C, Schlieper G, Nguyen P, et al: Serum fetuin-A levels are associated with vascular calcifications and predict cardiovascular events in renal transplant recipients. Clin J Am Soc Nephrol 2011;6:974-998.

7 Russo D, Morrone L, Russo L: Coronary artery calcification and cardiovascular mortality in predialysis patients. Kidney Int 2011;79: 258.

$\checkmark 8$ Matsuoka M, Iseki K, Tamashiro M, et al: Impact of high coronary artery calcification score (CACS) on survival in patients on chronic hemodialysis. Clin Exp Nephrol 2004;8:54-58.

9 Nguyen PT, Coche E, Goffin E, et al: Prevalence and determinants of coronary and aortic calcifications assessed by chest CT in renal transplant recipients. Am J Nephrol 2007;27: 329-335.

10 Arad Y, Goodman KJ, Roth M, Newstein D, Guerci AD: Coronary calcification, coronary disease risk factors, C-reactive protein, and atherosclerotic cardiovascular disease events: the St. Francis Heart Study. J Am Coll Cardiol 2005;46:158-165.

11 London GM: Cardiovascular calcifications in uremic patients: clinical impact on cardiovascular function. J Am Soc Nephrol 2003; 14(suppl 4):S305-S309.
12 Budoff MJ, Achenbach S, Blumenthal RS, et al: Assessment of coronary artery disease by cardiac computed tomography: a scientific statement from the American Heart Association Committee on Cardiovascular Imaging and Intervention, Council on Cardiovascular Radiology and Intervention, and Committee on Cardiac Imaging, Council on Clinical Cardiology. Circulation 2006;114:1761-1791.

13 Jacobs PC, Prokop M, van der Graaf Y, et al: Comparing coronary artery calcium and thoracic aorta calcium for prediction of all-cause mortality and cardiovascular events on lowdose non-gated computed tomography in a high-risk population of heavy smokers. Atherosclerosis 2010;209:455-462.

14 Raggi P, Bellasi A, Gamboa C, et al: All-cause mortality in hemodialysis patients with heart valve calcification. Clin J Am Soc Nephrol 2011;6:1990-1995.

15 Oschatz E, Benesch T, Kodras K, Hoffmann U, Haas M: Changes of coronary calcification after kidney transplantation. Am J Kidney Dis 2006;48:307-313.

16 Bargnoux AS, Dupuy AM, Garrigue V, et al: Evolution of coronary artery calcifications following kidney transplantation: relationship with osteoprotegerin levels. Am J Transplant 2009;9:2571-2579.

17 Mazzaferro S, Pasquali M, Taggi F, et al: Progression of coronary artery calcification in renal transplantation and the role of secondary hyperparathyroidism and inflammation. Clin J Am Soc Nephrol 2009;4:685-690.

18 Seyahi N, Cebi D, Altiparmak MR, et al: Progression of coronary artery calcification in renal transplant recipients. Nephrol Dial Transplant 2012;27:2101-2107.

19 Maréchal C, Coche E, Goffin E, et al: Progression of coronary artery calcification and thoracic aorta calcification in kidney transplant recipients. Am J Kidney Dis 2012;59:258-269.

20 Min JK, Lin FY, Gidseg DS, et al: Determinants of coronary calcium conversion among patients with a normal coronary calcium scan: what is the 'warranty period' for remaining normal? J Am Coll Cardiol 2010;55:11101117.

21 Kronmal RA, McClelland RL, Detrano R, et al: Risk factors for the progression of coronary artery calcification in asymptomatic subjects: results from the Multi-Ethnic Study of Atherosclerosis (MESA). Circulation 2007;115: 2722-2730.

22 McCullough PA, Chinnaiyan KM: Annual progression of coronary calcification in trials of preventive therapies: a systematic review. Arch Intern Med 2009;169:2064-2070.

23 Schankel K, Robinson J, Bloom RD, et al: Determinants of coronary artery calcification progression in renal transplant recipients. Am J Transplant 2007;7:2158-2164.

24 DeLoach SS, Joffe MM, Mai X, Goral S, Rosas SE: Aortic calcification predicts cardiovascular events and all-cause mortality in renal transplantation. Nephrol Dial Transplant 2009;24:1314-1319.

25 Moe SM, O’Neill KD, Reslerova M, Fineberg N, Persohn S, Meyer CA: Natural history of vascular calcification in dialysis and transplant patients. Nephrol Dial Transplant 2004; 19:2387-2393.

26 Yamamoto H, Shavelle D, Takasu J, et al: Valvular and thoracic aortic calcium as a marker of the extent and severity of angiographic coronary artery disease. Am Heart J 2003;146: 153-159.

27 Watanabe K, Hiroki T, Koga N: Relation of thoracic aorta calcification on computed tomography and coronary risk factors to obstructive coronary artery disease on angiography. Angiology 2003;54:433-441.

28 Adler Y, Fisman EZ, Shemesh J: Spiral computed tomography evidence of close correlation between coronary and thoracic aorta calcifications. Atherosclerosis 2004;176:133-138.

29 Mazzaferro S, Pasquali M, Pugliese F, et al: Serum levels of calcification inhibition proteins and coronary artery calcium score: comparison between transplantation and dialysis. Am J Nephrol 2007;27:75-83.

30 Rosas SE, Mensah K, Weinstein RB, Bellamy SL, Rader DJ: Coronary artery calcification in renal transplant recipients. Am J Transplant 2005;5:1942-1947.

-31 Mehrotra R, Budoff M, Hokanson JE, Ipp E, Takasu J, Adler S: Progression of coronary artery calcification in diabetics with and without chronic kidney disease. Kidney Int 2005; 68:1258-1266. 
32 Metry G, Stenvinkel P, Qureshi AR, et al: Low serum fetuin-A concentration predicts poor outcome only in the presence of inflammation in prevalent haemodialysis patients. Eur J Clin Invest 2008;38:804-811.

- 33 Urbanová M, Kalousová M, Zima T, Skibová J, Wohlfahrt P, Viklický O: Fetuin-A early after renal transplantation. Kidney Blood Press Res 2009;32:217-222.

- 34 London GM, Guérin AP, Verbeke FH, et al: Mineral metabolism and arterial functions in end-stage renal disease: potential role of 25-hydroxyvitamin D deficiency. J Am Soc Nephrol 2007;18:613-620.

- 35 Chitalia N, Recio-Mayoral A, Kaski JC, Banerjee D: Vitamin D deficiency and endothelial dysfunction in non-dialysis chronic kidney disease patients. Atherosclerosis 2012; 220:265-268.

- 36 Adeney KL, Siscovick DS, Ix JH, et al: Association of serum phosphate with vascular and valvular calcification in moderate CKD. J Am Soc Nephrol 2009;20:381-387.

-37 Raggi P, Boulay A, Chasan-Taber S, et al: Cardiac calcification in adult hemodialysis patients. A link between end-stage renal disease and cardiovascular disease? J Am Coll Cardiol 2002;39:695-701.

38 Burger D, Levin A: 'Shedding' light on mechanisms of hyperphosphatemic vascular dysfunction. Kidney Int 2013;83:187-189.

- 39 Coll B, Betriu A, Martínez-Alonso M, et al: Large artery calcification on dialysis patients is located in the intima and related to atherosclerosis. Clin J Am Soc Nephrol 2011;6:303310.

-40 Connolly GM, Cunningham R, McNamee PT, Young IS, Maxwell AP: Elevated serum phosphate predicts mortality in renal transplant recipients. Transplantation 2009;87: 1040-1044.

- 41 Isakova T, Xie H, Yang W, et al: Fibroblast growth factor 23 and risks of mortality and end-stage renal disease in patients with chronic kidney disease. JAMA 2011;305: 2432-2439.

-42 Khan AM, Chirinos JA, Litt H, Yang W, Rosas SE: FGF-23 and the progression of coronary arterial calcification in patients new to dialysis. Clin J Am Soc Nephrol 2012;7:2017-2022.

43 Sánchez Fructuoso AI, Maestro ML, PérezFlores I, et al: Serum level of fibroblast growth factor 23 in maintenance renal transplant patients. Nephrol Dial Transplant 2012;27: 4227-4235.
44 Wolf M, Molnar MZ, Amaral AP, et al: Elevated fibroblast growth factor 23 is a risk factor for kidney transplant loss and mortality. J Am Soc Nephrol 2011;22:956-966.

45 Moe SM, Reslerova M, Ketteler M, et al: Role of calcification inhibitors in the pathogenesis of vascular calcification in chronic kidney disease. Kidney Int 2005;67:2295-2304.

46 Yoshikawa K, Abe H, Tominaga T, et al: Polymorphism in the human matrix Gla protein gene is associated with the progression of vascular calcification in maintenance hemodialysis patients. Clin Exp Nephrol 2013;17:882889.

47 Rennenberg RJ, de Leeuw PW, Kessels AG, et al: Calcium scores and matrix Gla protein levels: association with vitamin $\mathrm{K}$ status. Eur J Clin Invest 2010;40:344-349.

48 Boxma PJ, Van der Berge E, Geleijnse JM, et al: Vitamin K intake and plasma desphosphoundercarboxylated matrix Gla protein in kidney transplant recipients. PLoS One 2012;7: e47991.

49 Morena M, Terrier N, Jaussent I, et al: Plasma osteoprotegerin is associated with mortality in hemodialysis patients. J Am Soc Nephrol 2006; 17:262-270.

-50 Svensson M, Dahle DO, Mjøen G, et al: Osteoprotegerin as a predictor of renal and cardiovascular outcomes in renal transplant recipients: follow-up data from the ALERT study. Nephrol Dial Transplant 2012;27: 2571-2575.

51 Budoff MJ, Yu D, Nasir K, et al: Diabetes and progression of coronary calcium under the influence of statin therapy. Am Heart J 2005; 149:695-700.

52 Snell-Bergeon JK, Hokanson JE, Jensen L, et al: Progression of coronary artery calcification in type 1 diabetes: The importance of glycemic control. Diabetes Care 2003;26:29232928.

53 Chen NX, Duan D, O'Neill KD, Moe SM: High glucose increases the expression of Cbfa1 and BMP-2 and enhances the calcification of vascular smooth muscle cells. Nephrol Dial Transplant 2006;21:3435-3442.

54 Nickel T, Schlichting CL, Weis M: Drugs modulating endothelial function after transplantation. Transplantation 2006;82(suppl 1):S41-S46

55 Shimizu H, Takahashi M, Takeda S, et al: Mycophenolate mofetil prevents transplant arteriosclerosis by direct inhibition of vascular smooth muscle cell proliferation. Transplantation 2004;77:1661-1667.
56 Westenfeld R, Schlieper G, Wöltje M, et al: Impact of sirolimus, tacrolimus and mycophenolate mofetil on osteoclastogenesis - implications for post-transplantation bone disease. Nephrol Dial Transplant. 2011;26:41154123.

$57 \mathrm{Wu}$ B, Elmariah S, Kaplan FS, Cheng G, Mohler ER III: Paradoxical effects of statins on aortic valve myofibroblasts and osteoblasts: implications for end-stage valvular heart disease. Arterioscler Thromb Vasc Biol 2005;25:592-597.

$58 \mathrm{He}$ ZX, Hedrick TD, Pratt CM, et al: Severity of coronary artery calcification by electron beam computed tomography predicts silent myocardial ischemia. Circulation 2000;101: 244-251.

59 Detrano R, Guerci AD, Carr JJ, et al: Coronary calcium as a predictor of coronary events in four racial or ethnic groups. N Engl J Med 2008;358:1336-1345

60 Greenland P, LaBree L, Azen SP, Doherty TM, Detrano RC: Coronary artery calcium score combined with Framingham score for risk prediction in asymptomatic individuals. JAMA 2004;291:210-215.

61 Kidney Disease: Improving Global Outcomes (KDIGO) Transplant Work Group: KDIGO clinical practice guideline for the care of kidney transplant recipients. Am J Transplant 2009;9(suppl 3):S1-S155.

62 Lentine KL, Costa SP, Weir MR, et al: Cardiac disease evaluation and management among kidney and liver transplantation candidates: a scientific statement from the American Heart Association and the American College of Cardiology Foundation. Circulation 2012;126: 617-663.

63 Degoma EM, Dunbar LM, Jacoby D, French B: Differences in absolute risk of cardiovascular events using risk-refinement tests: a systematic analysis of four cardiovascular risk equations. Atherosclerosis 2013;227:172-177.

64 Nguyen PT, Henrard S, Coche E, et al: Coronary artery calcification: a strong predictor of cardiovascular events in renal transplant recipients. Nephrol Dial Transplant 2010;25:3773-3778.

65 Roe P, Wolfe M, Joffe M, Rosas S: Inflammation, coronary artery calcification and cardiovascular events in incident renal transplant recipients. Atherosclerosis 2010;212:589594 